\title{
Right Dorsolateral Prefrontal Cortex Activation during a Time Production Task: A Functional Near-Infrared Spectroscopy Study
}

\author{
Asato Morita, ${ }^{1}$ Yasunori Morishima, ${ }^{2}$ and David W. Rackham ${ }^{2}$ \\ ${ }^{1}$ Graduate School of Arts and Sciences, International Christian University, Tokyo 181-8585, Japan \\ ${ }^{2}$ Department of Psychology, International Christian University, Tokyo 181-8585, Japan \\ Correspondence should be addressed to Asato Morita; asamorita@gmail.com
}

Received 17 January 2015; Revised 12 April 2015; Accepted 30 April 2015

Academic Editor: Jinsung Wang

Copyright (c) 2015 Asato Morita et al. This is an open access article distributed under the Creative Commons Attribution License, which permits unrestricted use, distribution, and reproduction in any medium, provided the original work is properly cited.

\begin{abstract}
Accurate time estimation is crucial for many human activities and necessitates the use of working memory, in which the dorsolateral prefrontal cortex (DLPFC) plays a critical role. We tested the hypothesis that the DLPFC is activated in participants attempting time estimations that require working memory. Specifically, we used functional near-infrared spectroscopy (fNIRS) to investigate prefrontal cortical activity in the brains of individuals performing a prospective time production task. We measured cerebral hemodynamic responses in 26 healthy right-handed university students while they marked the passage of specified time intervals $(3,6,9,12$, or $15 \mathrm{~s})$ or performed a button-pressing (control) task. The behavioral results indicated that participants' time estimations were accurate with minimal variability. The fNIRS data showed that activity was significantly higher in the right DLPFC during the time estimation task compared to the control task. Theoretical considerations and the results of this study suggest that DLPFC activation resulting from time estimation indicates that the working memory system is in use.
\end{abstract}

\section{Introduction}

The ability to estimate time plays an important role in adaptation to the environment. For example, when a student takes a test, accurate estimation of time intervals is necessary to efficiently solve many problems within the time limit. Specifically, time estimation refers to appraising temporal duration without using information from a clock. Whereas the number of time estimation studies has increased in recent years [1], the underlying mechanisms remain unclear. Time estimation in the range of seconds to minutes is considered to be controlled by diverse cognitive systems [2], whereas time estimation in the millisecond range is thought to be associated with the motor system [3]. As our interest is accurate time estimation in daily activities, we focused on the biological substrates and cognitive systems of time estimation in the range of seconds.

Several models have been developed to account for time estimation $[4,5]$. The scalar expectancy theory (SET) is the most popular model of time estimation (see Figure 1) $[6,7]$.
It posits that time estimation is based on three informationprocessing stages: clock, memory, and decision [6]. In the SET, a hypothetical internal pacemaker emits pulses that are gated by a switch during the current to-be-timed interval and then sent to an accumulator [7]. The content in the accumulator (number of pulses) corresponds to the current time. The accumulator's content is transferred and stored in the working memory, which is compared with learned time labels for known intervals previously stored in the reference memory. The comparison between these accumulated pulses in working memory and learned temporal representations in reference memory determines the time estimation response. According to this model, individual differences in time estimation may be attributable to alterations in pacemaker speed, memory efficiency, and comparator function [5]. Despite the effectiveness of the SET in explaining various behavioral and physiological results, its relevance to the neural substrates involved in accurate time estimation is not fully clear [8]. Many lines of evidence suggest that separate brain mechanisms are responsible for different stages of 


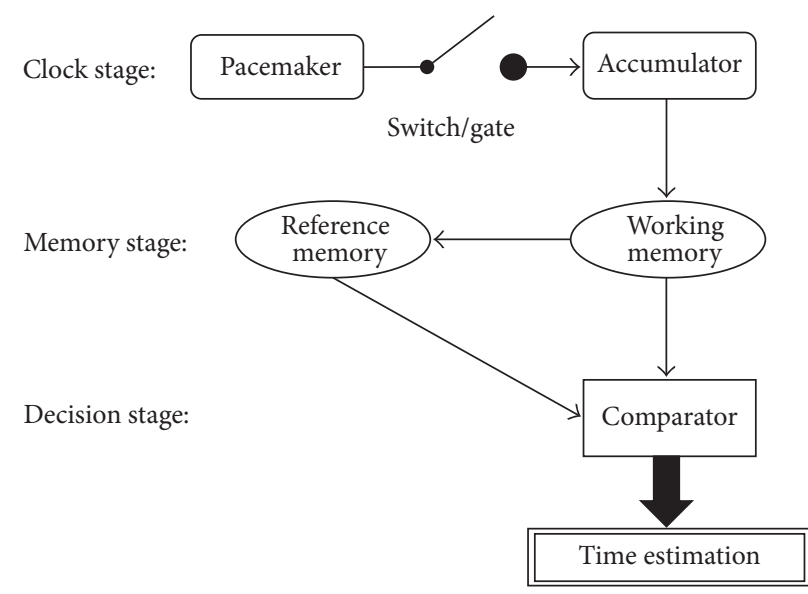

FIGURE 1: Scalar expectancy theory (SET) applied to the time production task. In SET, a pacemaker emits pulses to an accumulator, and the number of pulses is transferred to the working memory by way of an accumulator. The corresponding number of pulses is stored in the working memory and compared with that of the reference intervals stored in reference memory. When the numbers of pulses match, the participant responds.

the SET [8]. In this framework, a memory stage is functionally separated from other processing stages [9], and accurate time estimation capacity is heavily dependent on working memory efficiency.

Several recent reviews and meta-analyses of neuroimaging studies have shown that many parts of the brain contribute to time estimation. Macar and colleagues [10] defined the dorsolateral prefrontal cortex (DLPFC), anterior cingulate, right inferior parietal lobe (IPL), supplementary motor area (SMA), cerebellum, and basal ganglia (caudate and putamen) as the core time estimation network. Lewis and Miall [11] reviewed many neuroimaging studies of timing and concluded that suprasecond timing tasks most commonly activated the bilateral prefrontal cortex, bilateral parietal cortex, and cerebellum. In their studies, the right DLPFC was the most frequently activated area. In contrast, a relatively recent meta-analysis reported that the SMA and right inferior frontal gyrus were part of the core network mediating time estimation in the brain, whereas the DLPFC was less important for time estimation [1]. Thus, there have been inconsistencies regarding the neural correlates of time estimation in previous studies, probably because different brain structures are activated depending on the paradigm, temporal task, and duration range used $[1,3]$.

Most studies have used functional magnetic resonance imaging (fMRI) to examine brain activity during time estimation, as it can noninvasively measure brain activity, does not involve radiation, and has high spatial resolution. However, several shortcomings are associated with fMRI, including confined spaces, scanner noise, and the loss of situational control experienced by the participant, which can elicit anxiety and stress (e.g., [12]) that in turn could affect behavioral and neurological data [13]. In addition to these disadvantages of fMRI, the time estimation may also be affected by the difference in the body posture. However, the actual effect of posture on time estimation is unknown. During fMRI scanning, participants lie in a supine position, whereas time estimation experiments using behavioral techniques are usually performed while the participant is sitting upright in front of a monitor [14]. Muehlhan and colleagues [14] investigated the effect of body posture on cognitive performance, and their results indicate that sleep quality strongly affected reaction times when participants performed a working memory task in the supine posture, but these effects were not observed in the sitting position. It has also been reported that differences in orthostatic load between sitting and supine positions lead to physiological changes [14-16]. Additionally, there are beneficial effects of the sitting position over the supine position on cognitive performance [14].

In the present study, we employed functional near-infrared spectroscopy (fNIRS) to investigate prefrontal activity during the time production task. This noninvasive neuroimaging technique enables the measurement of relative changes in concentrations of oxygenated and deoxygenated hemoglobin (oxy- and deoxy-Hb, resp.) in the superficial layer of the cortex [17]. Although fNIRS can only record at the brain surface and has low spatial resolution $(3 \mathrm{~cm})$, it can tolerate movement and is suitable for use in seated participants [18].

Neurologically normal adults are relatively accurate in estimating time (e.g., $[19,20])$. We were interested in participants' capacity for accurate time estimation of conventional duration units (i.e., we wanted to determine how accurately a student estimates 15 seconds), for which there are few studies. Various tasks have been employed to investigate individuals' time estimation. The present study employed a prospective time production task to quantify time estimation; this methodology is widely used [21] because the participants' load is minimal, and the experimental procedure is easy. This task is suitable for our research objective because it relies on the scaling of subjective time by units used in daily life. In the time production task, participants are asked to indicate when a stated time has elapsed. In particular, the task requires the participants to mark the "start" and "stop" when they thought an identified time period had passed. We used the prospective time estimation paradigm [22] where participants know in advance that they will be asked to produce a given time duration. The control task was pressing a button twice in a manner similar to the time production task, but without a specified target time. This task was equivalent to the motor requirement of the time production task. We consider that accurately producing a particular duration depends on the memory stage of the SET; the participant should make a comparison between accumulated pulses in working memory and a learned temporal representation of reference memory.

When participants must accurately produce target duration in conventional time units, the SET $[6,7]$ would predict that they compare the content of time units accumulated and stored in working memory with the representation of time stored in reference memory. Working memory is important for a wide range of high-level cognitive activities and is commonly defined as the system used to temporarily store information and then manipulate the information online during cognitive activities [23]. Previous studies have shown that 
the DLPFC plays a critical role in working memory. Patients with traumatic brain injury or Parkinson's disease generally show working memory deficits and inaccurate time estimation [3]. Working memory capacity is thought to be indispensable for time estimation, as the frontal lobe would be needed to store the current interval in working memory, recall a sense of time in reference memory, and compare both values.

The present study compared fNIRS-measured frontal cortex activity during the time production task with that measured during the control task. Specifically, we tested the hypothesis that the DLPFC would be activated in participants attempting time estimations that required the working memory.

This study was designed to assess the role of the working memory system and identify the brain structures involved in accurate time estimation. We intended to separate the memory and decision-stage functions from clock-stage function, which merely predicts the time interval by comparing the time production and button-pressing tasks. The time production task uses clock, memory, and decision stages that rely on both working and reference memories [3], whereas the button-pressing task does not require memory processes. The results were interpreted within the SET theoretical framework. To our knowledge, this is the first report of the assessment of prefrontal activity using fNIRS during a time production task.

\section{Materials and Methods}

2.1. Participants. Twenty-six right-handed, healthy volunteers $(7$ males and 19 females, mean age $=20.58$ years, $\mathrm{SD}=$ 2.00, and range: 19-27 years) participated in the study. Handedness was assessed using the Edinburgh Handedness Inventory [24]. All participants provided written informed consent prior to participating in the experiment, for which they received a coupon worth 500 Japanese yen at the end of the experiment. This study was conducted in accordance with the Declaration of Helsinki [25] and was approved by the relevant ethics committee.

2.2. Experimental Design. The design of the fNIRS experiment was a simple block design with one experimental and one control task condition. The 26 participants were divided into 2 groups of 13 participants. Each group was then assigned to one of two patterns to balance the two conditions (Figure 2). Cerebral activations measured with fNIRS were then compared between the two conditions. Each condition was repeated three times in a predetermined pattern; thus, the experiments consisted of six blocks with a $60 \mathrm{~s}$ break between each block (Figure 2). The time production task block consisted of five trials, each of which contained the five intervals $(3,6,9,12$, and $15 \mathrm{~s})$. The order of trial presentation was counterbalanced across participants. The button-pressing task consisted of five trials to match the time interval required for the time production task. There was a $500 \mathrm{~ms}$ interval when the cue was displayed on the screen between each trial (i.e., between the participant response and

\begin{tabular}{|c|c|}
\hline Pattern A & Pattern B \\
\hline Start & Start \\
\hline Rest: $30 \mathrm{~s}$ & Rest: $30 \mathrm{~s}$ \\
\hline (1) Time production task & (1) Button-pressing task \\
\hline Rest: $60 \mathrm{~s}$ & Rest: $60 \mathrm{~s}$ \\
\hline (2) Button-pressing task & (2) Time production task \\
\hline Rest: $60 \mathrm{~s}$ & Rest: $60 \mathrm{~s}$ \\
\hline (3) Button-pressing task & (3) Time production task \\
\hline Rest: $60 \mathrm{~s}$ & Rest: $60 \mathrm{~s}$ \\
\hline (4) Time production task & (4) Button-pressing task \\
\hline R $\quad$ Rest: $60 \mathrm{~s}$ & Rest: $60 \mathrm{~s}$ \\
\hline (5) Time production task & (5) Button-pressing task \\
\hline$\sqrt{2}$ Rest: $60 \mathrm{~s}$ & $\checkmark \quad$ Rest: $60 \mathrm{~s}$ \\
\hline (6) Button-pressing task & (6) Time production task \\
\hline 2 $\quad$ Rest: $30 \mathrm{~s}$ & Rest: $30 \mathrm{~s}$ \\
\hline End & End \\
\hline
\end{tabular}

FIGURE 2: Schematic representation of the two sets of instruction sequences (Patterns $\mathrm{A}$ and $\mathrm{B}$ ) that comprised the estimation of time duration test. All participants were assigned to one of two groups. Each group was then assigned to one of two patterns to balance the two conditions. Each condition was repeated three times in a predetermined pattern; thus, the experiments consisted of six blocks, with a $60 \mathrm{~s}$ break between blocks. The time production task consisted of marking the start and end of perceived times of specified intervals by pressing a button on the response box. The intervals ( 3 , $6,9,12$, or $15 \mathrm{~s}$ ) were randomized for each participant. The buttonpressing task was a control task.

the onset of the next trial (Figure 3)). The participants did not receive any feedback.

2.3. Apparatus and Stimuli. The experiment was programmed and run using SuperLab Pro 4.5 for Windows, with a Cedrus RB-540 response box used to record the participant responses (Cedrus Corporation, San Pedro, CA). For all tasks, the stimuli were presented on a laptop computer (Let's NOTE CF-R5, Panasonic Corporation, Osaka, Japan) with a display area of $21.1 \times 15.8 \mathrm{~cm}$ and a screen resolution of $1024 \times 768$ pixels. The words were presented in the center of the computer screen in black MS Gothic 48-point font on a white background. The distance from the laptop screen to the participant's head was approximately $60 \mathrm{~cm}$. A second laptop computer (VOSTRO-3750, Dell Inc., Round Rock, TX) was used to record and analyze fNIRS data.

2.4. Time Estimation and Control Tasks. The time production and button-pressing task protocols are shown in Figure 2. The experiment session began with $30 \mathrm{~s}$ of rest (no body movement). Subsequently, a fixation cross was displayed, and an auditory cue (a pure sine wave of $800 \mathrm{~Hz}$ ) was played for $100 \mathrm{~ms}$ between trials to arouse attention. Then, the instructions were given to the participant. A marking stimulus was synchronously presented at the start and end of each trial and externally fed into the fNIRS device.

In the time production task, participants were instructed to subjectively estimate the presented length of time. The specified length of time to produce was displayed on the horizontal axis of the computer monitor for $1700 \mathrm{~ms}$. The screen 


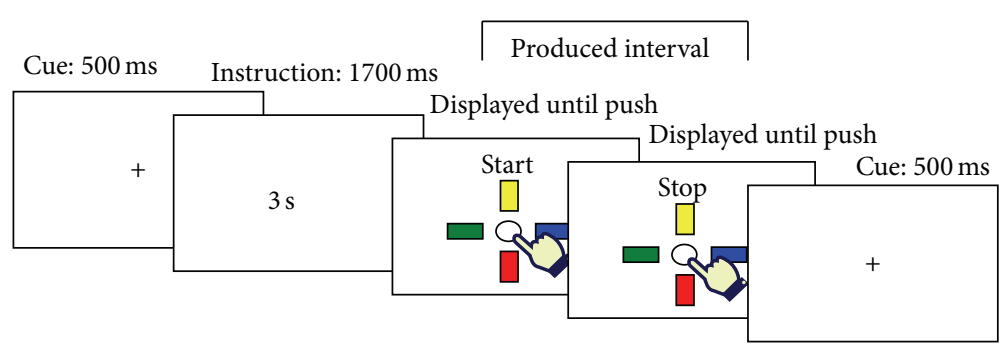

(a) Time production task

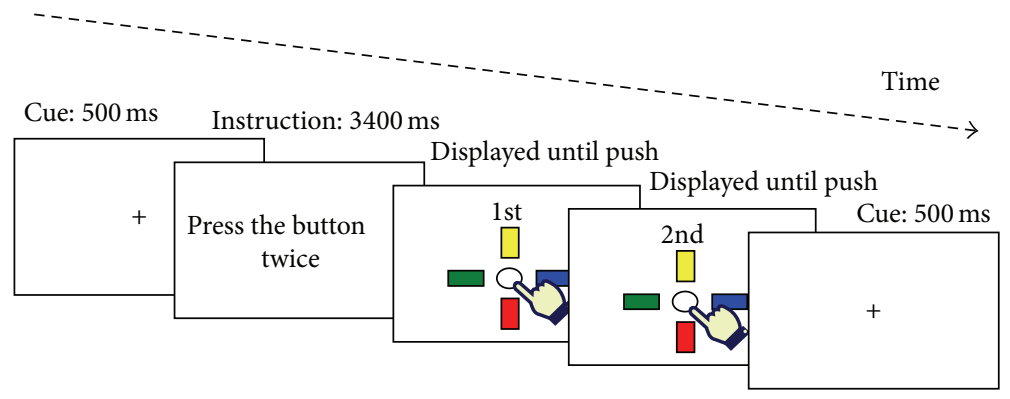

(b) Button-pressing task

Figure 3: Sequences of events during the time production and button-pressing tasks. (a) Time production task. The number of seconds to be produced was shown on the screen, and time production began and ended with the participant's first and second button press, respectively. All instructions were given in Japanese. (b) Button-pressing task. In contrast to the time production task, the time instructions were not displayed. Rather, the following instructions were shown in Japanese: "Press the button twice."

then changed to display "Start" and the participant pressed the button in the response box to begin time production. When the participant felt that the specified length of time had passed, the participant again pressed the button in the response box. The sum of the time perception intervals was $45 \mathrm{~s}$. Summing the five $1700 \mathrm{~ms}$ instructed delay cues and the delays associated with starting the task resulted in a time production interval that should last at least $55 \mathrm{~s}$.

In the button-pressing task, we asked participants to press the button twice; this task's motion was equivalent to that of the time production task. The length of the interval between the button presses was not specified by the experimenter. However, a gap of a certain duration existed between the first and second presses, and we could adjust the length of the block duration. Participants were asked to press the button twice at their own preferable interval, but without an interval that was too short and without overthinking. The task began with a $3400 \mathrm{~ms}$ display of instructions to "press the button twice at a random duration, where the duration is not too short, without overthinking." The participants were also told to perform this task in a manner that was comfortable. The control task involved a $3400 \mathrm{~ms}$ display of the instructions; coupled with the associated intertrial delays and movement delays, it was expected to last approximately $40 \mathrm{~s}$.

2.5. fNIRS Instruments. A multichannel fNIRS system (OEG-16, Spectratech Inc., Tokyo, Japan) equipped with six near-infrared light sources and six detectors was used to monitor changes in oxy-Hb concentrations in 16 channels (Figure 4). For each channel, the absorption of near-infrared

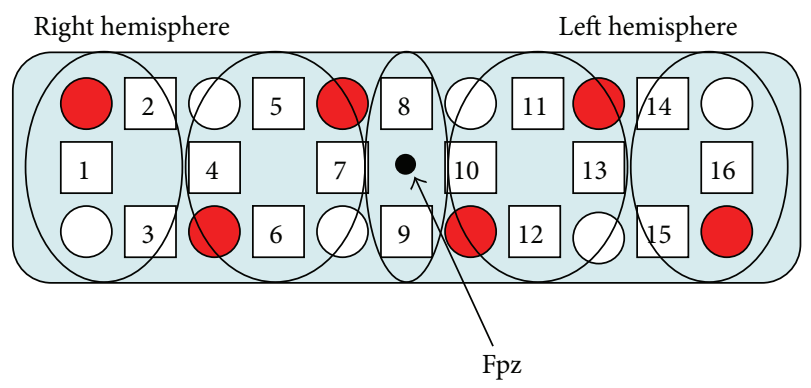

FIgURE 4: Arrangement of incident, detection, and measurement positions (channels). Cortical responses were obtained from 16 locations. The center of the probe matrix was placed at Fpz (the midpoint between Fp1 and Fp2) in accordance with the International 10-20 system. Red, light emitter; white, light receiver; number, channel number.

light at 770 and $840 \mathrm{~nm}$ was measured at a scanning rate of $650 \mathrm{~ms}$.

2.6. fNIRS Channel Positions. Each channel was constructed by a pair of emitter and detector probes at a distance of $3 \mathrm{~cm}$ from each other. The forehead region under measurement was $3 \mathrm{~cm}$ long and $15 \mathrm{~cm}$ wide, and sensor placement was in accordance with the Fpz standard of the International 10-20 system [26]. All 16 channels were used for data collection. Figure 4 indicates the types of arrays and landmarks.

2.7. Experimental Procedures. Each participant performed the tasks while sitting on a comfortable chair in a dimly lit 
silent room. Participants were asked to sign a consent form indicating their willingness to participate in the experiment after the experimenter had provided them with a general description of the purpose of the experiment. Next, the head circumference, the distance from nasion to inion, and Fpz of the participants were measured. Participants were then fitted with the fNIRS probe headband and given a brief practice session to ensure that they understood the two tasks. After relatively stable $\mathrm{Hb}$ signals were confirmed, the time production or button-pressing task was started. We examined hemodynamic changes in the prefrontal cortex using fNIRS when participants were performing the time production and button-pressing tasks. All participants were then fully debriefed regarding the study's purpose and thanked for their time following experiment completion. The amount of time for the fNIRS measurement was approximately 15 minutes, and the whole experiment required approximately 30 minutes to complete.

2.8. Data Analysis. Two scores were derived from the time production task: the ratio scores and the coefficient of variance. These are classical indices of performance in timing studies (e.g., $[3,19,27])$. The accuracy and variability of time estimation can also vary according to the conditions in which the particular tasks or paradigms were used. The ratio score was calculated by taking the ratio of the duration estimated by the participants to the target duration, and it reflects the accuracy of the size of the standard interval $[3,19]$. A perfect estimation, according to the ratio score, would be 1.00, whereas scores below and above 1.00 reflect underestimations and overestimations, respectively. The coefficient of variance index was computed by taking the ratio of the standard deviation to the production mean and represents the variability of time estimation for each participant $[3,19,27]$. This index enabled the consistency of the participants in estimating the same target duration to be evaluated [3].

The fNIRS data were first preprocessed before the functional localization analyses, and then the relationship between the behavioral data and brain activity was examined. The relative hemoglobin concentration (oxy-Hb and deoxy$\mathrm{Hb}$ ) for each of the $16 \mathrm{fNIRS}$ channels was calculated using light signals transmitted at the two wavelengths on the basis of the modified Beer-Lambert law [28] and expressed as concentration (in $\mathrm{mM}$ ) per unit path length (in $\mathrm{mm}$ ) traversed by the near-infrared light through the brain surface $(\mathrm{mM} / \mathrm{mm})$. The exact differential path length factor was not measurable [18]. Raw hemoglobin data are measured as relative values that cannot be quantified and thus cannot be compared between participants or between channels within a given participant [29]. Oxy-Hb is highly correlated with changes in regional cerebral blood flow that reflect synaptic activity [30, 31]; therefore, only oxy-Hb was used in the analysis.

To avoid an increase in the familywise error rate, the 16 channels were divided into 5 areas. Channels 1-3 corresponded to the right DLPFC, channels 4-7 corresponded to the right medial frontal lobe, channels 8 and 9 corresponded to the frontal pole, channels 10-13 corresponded to the left medial frontal lobe, and channels 14-16 corresponded to the left DLPFC. The mean values for each of the five areas were used for all analyses. The data were stored in the fNIRS machine and further analyzed using OEG-16 software.

Although previous fNIRS studies have used various methods to detect motion artifacts, a standard method for this process remains to be established [32]. Some studies have employed subjective methods based on visual inspection [33]. In this study, sharp noises detected on visual inspection were regarded as motion artifacts.

The high-frequency portions of the signals were removed by calculating a moving average with a 4.55-s time window. Then, OEG-16 software was used to separately average signals in response to each trial across the three blocks for the time production and button-pressing tasks. To exclude slow drifts in the signals, a linear trend was removed from the data based on the mean baseline signals $10 \mathrm{~s}$ before and $30 \mathrm{~s}$ after the task blocks. The averaged oxy-Hb level in response to the time estimation and button-pressing tasks was exported by the OEG-16 software in CSV data format. The oxy-Hb data for a 30-s period from $5 \mathrm{~s}$ after stimulus onset was defined as a cerebral reaction change related to the task. Mean signal changes for 30-s periods were calculated for each participant for each channel. We wished to view each block of the time estimation task relative to each block of the button-pressing task. Therefore, we adjusted the block length in the control task to match the block length in the time estimation task. However, because it would be difficult for the participants to equalize the block length in the time production task with that in the button-pressing task, we did not use the entire time estimation period; rather, we chose to analyze the data from $5 \mathrm{~s}$ to $35 \mathrm{~s}$ of this period (the $5 \mathrm{~s}$ delay was caused by hemodynamic lag); another reason why only part of the block was used for analysis was that participants became habituated in the later part of the block.

2.9. Statistical Analysis. The ratio score and coefficient of variance index were compared across the five different time intervals $(3,6,9,12$, and $15 \mathrm{~s})$ to determine whether the averages for these intervals were significantly different. Separate one-way repeated measures analyses of variance (ANOVAs) were conducted for the ratio scores and coefficient of variance data using the time interval $(3,6,9,12$, and $15 \mathrm{~s}$ ) as a within-participant variable. The GreenhouseGeisser correction procedure was used to adjust the degrees of freedom when appropriate [34]. For these analyses, the alpha level for significance was set to 0.05 , and all post hoc tests were Bonferroni-corrected.

To determine whether significant changes in the oxy$\mathrm{Hb}$ signal occurred in the frontal cortex, paired Student's $t$ test (two-tailed) was used to compare the signal between the time estimation and button-pressing tasks. These statistical analyses were performed for five brain areas. In this analysis, the levels of significance were adjusted using the Bonferroni correction, which is regarded as conservative, as it involves dividing the alpha level by the number of brain areas $(0.05 / 5=$ 0.01). We conducted all statistical analyses using the Statistical Package for Social Sciences (SPSS) for Windows, Version 15.0 (SPSS Inc., Chicago, IL). 


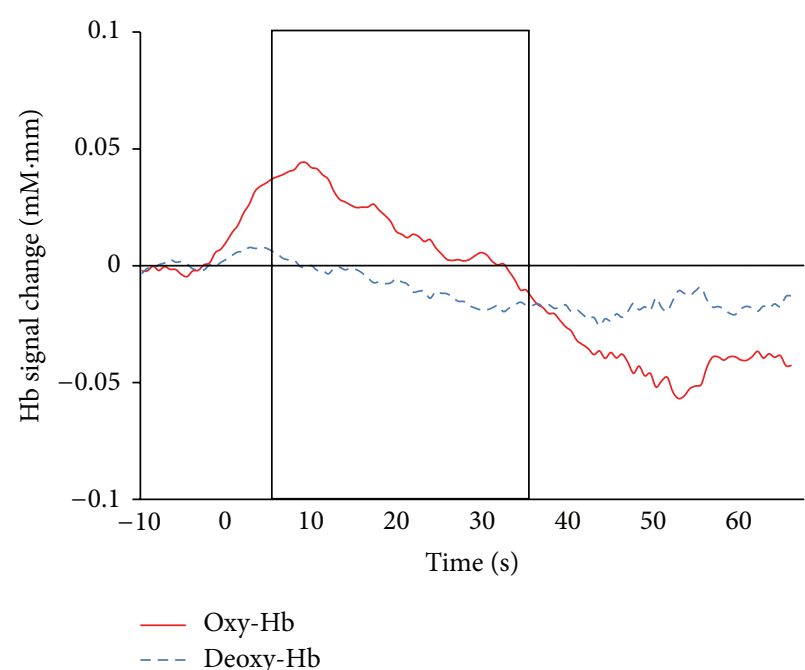

(a)

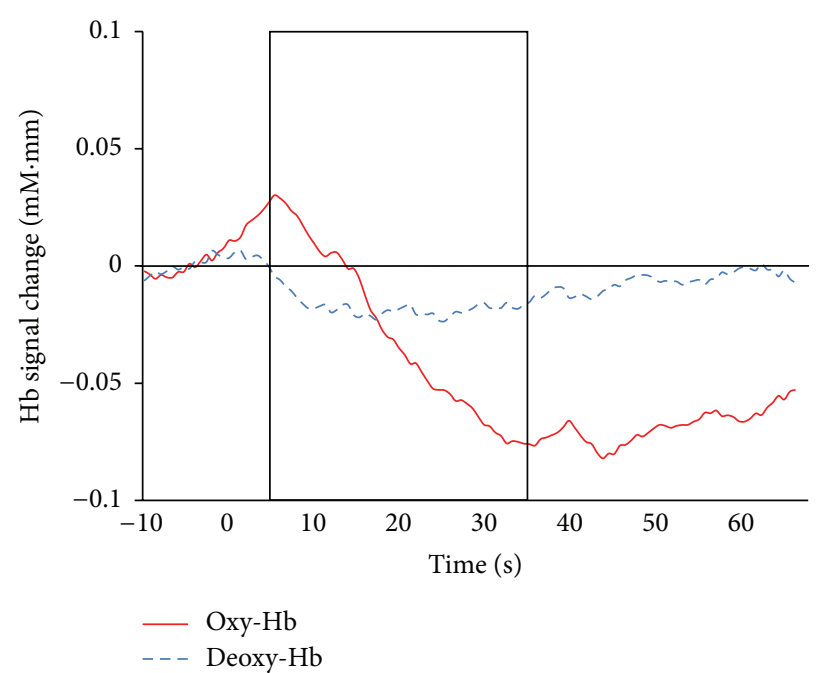

(b)

FIGURE 5: Time course for concentrations of oxy- and deoxy-hemoglobin in the right dorsolateral prefrontal cortex (DLPFC) during the (a) time production and (b) button-pressing tasks (control). fNIRS data recorded in channels 1-3 (corresponding to the right DLPFC) were filtered, and the averaged values of the (a) time production and (b) button-pressing tasks are plotted. The boxed area (the $30 \mathrm{~s}$ period from 5 to $35 \mathrm{~s}$ ) indicates the data used in the analyses.

TABLE 1: Demographic data (means and standard deviations).

\begin{tabular}{lcccccc}
\hline Time & \multicolumn{3}{c}{ Raw score } & \multicolumn{2}{c}{ Ratio score } & \multicolumn{2}{c}{ Coefficient of variance } \\
intervals & Mean & SD & Mean & SD & Mean & SD \\
\hline $3 \mathrm{~s}$ & 3.04 & 0.91 & 1.01 & 0.30 & 0.11 & 0.12 \\
$6 \mathrm{~s}$ & 6.34 & 1.29 & 1.06 & 0.22 & 0.07 & 0.04 \\
$9 \mathrm{~s}$ & 9.69 & 1.66 & 1.08 & 0.18 & 0.05 & 0.03 \\
$12 \mathrm{~s}$ & 13.06 & 2.09 & 1.09 & 0.17 & 0.04 & 0.03 \\
$15 \mathrm{~s}$ & 16.14 & 2.34 & 1.08 & 0.16 & 0.07 & 0.13 \\
\hline
\end{tabular}

\section{Results}

3.1. Behavioral Data. Table 1 shows the mean time required to estimate the different intervals in the time estimation task. Differences in the ratio score and coefficient of variance index among the different time estimation intervals were examined. A one-way repeated measures ANOVA for the ratio score of time production did not reveal a significant difference among the intervals, $F(1.69,42.26)=2.71$, MSE $=0.019, p=0.086$, partial $\eta^{2}=0.098$. The ANOVA for the coefficient of variance index revealed that response consistency did not differ among the five intervals, $F(1.99,49.65)=2.73, \mathrm{MSE}=0.011, p=0.076$, partial $\eta^{2}=0.098$.

As stated above, the indices of accuracy and response consistency remained almost identical among the five intervals. Hence, we did not classify the five intervals separately based on the fNIRS data analysis; rather, we averaged them as a single overall time estimation condition.

3.2. fNIRS Data. The difference in prefrontal cortex activation was evaluated in terms of oxy-Hb changes during the time estimation and button-pressing tasks. Paired-samples $t$-tests were performed to compare the fNIRS data for each channel recorded during the two tasks (Table 2). In channels 1-3 (right DLPFC), the mean relative change in the concentration of oxy-Hb during the time production task was significantly higher than that during the button-pressing task, $t(25)=2.896$, corrected $p=0.040, d_{\mathrm{D}}=0.57$ (Figure 5). As shown in Table 2, there were no significant differences in the mean relative change in the concentration of oxy- $\mathrm{Hb}$ between the time production and button-pressing tasks in other positions $(p>0.05)$.

\section{Discussion}

The present study used fNIRS to examine activity in the frontal brain regions associated with the accurate estimation of time using conventional time units. We compared the frontal cortex activities of participants estimating a specified time interval with those measured during the control task. To focus on time estimation accuracy in daily life, we instructed the participants to produce a particular duration using conventional time units (e.g., seconds). The protocol was designed to test the hypothesis that working memory is required to accurately estimate time, that is, that the DLPFC would be active during the time production task.

The mean hemodynamic response of oxy-Hb in the right DLPFC during the time estimation task was higher than that during the control task, which supports our hypothesis. As noted in the introduction, we compared the time production and button-pressing tasks to separate memory and decisionstage functions from the clock-stage function that is responsible for estimation of time intervals. In particular, we sought to assess the roles of the working memory and specific frontal cortex structures involved in accurate time estimation. We could not directly measure reference memory, as the activated 
TABLE 2: Oxy-Hb activity of time production task and button-pressing task.

\begin{tabular}{|c|c|c|c|c|c|c|}
\hline \multirow{2}{*}{ Position } & \multicolumn{2}{|c|}{ Time production task } & \multicolumn{2}{|c|}{ Button-pressing task } & \multirow{2}{*}{$t$ value } & \multirow{2}{*}{$p$ value (corrected) } \\
\hline & Mean & $\mathrm{SD}$ & Mean & SD & & \\
\hline ch1-ch3 & 0.020 & 0.077 & -0.027 & 0.051 & 2.896 & 0.040 \\
\hline $\operatorname{ch} 4-\operatorname{ch} 7$ & -0.021 & 0.093 & -0.052 & 0.051 & 1.768 & 0.445 \\
\hline ch8-ch9 & -0.058 & 0.108 & -0.082 & 0.059 & 1.243 & 0.999 \\
\hline $\operatorname{ch} 10-\operatorname{ch} 13$ & -0.037 & 0.098 & -0.058 & 0.056 & 1.104 & 0.999 \\
\hline ch14-ch16 & 0.009 & 0.086 & -0.027 & 0.051 & 2.243 & 0.170 \\
\hline
\end{tabular}

Note. ch: channel.

The different channels (ch) correspond to different regions of the prefrontal cortex as follows: ch1 to ch3, right dorsolateral prefrontal cortex (DLPFC); ch4 to ch7, right medial frontal lobe; ch8 and ch9, central regions including the frontal pole; ch10 to ch13, left medial frontal lobe; and ch14 to ch16, left DLPFC.

area is subcortical $[3,9]$, and the fNIRS instrument used in the present study could only measure the frontal cortex.

Theoretically, both working and reference memories would be required for the time production task [8], which in contrast to the control task requires associating a given duration with a representation of intervals or knowledge of conventional time units [3]. The representation of conventional time units is stored in the reference memory [3]. According to Perbal-Hatif [3], reference memory is assimilated with semantic memory, which stores general knowledge of the world including time representation. In the present task, first, participants had to maintain a target duration given in conventional units of time in their mind until the trial was over. Then, the participants had to retrieve information based on previous experience from their reference memory and simultaneously monitor the experienced duration using the working memory system. The participants had to continually compare the elapsing duration in the working memory to representations stored as the target duration in the reference memory. Thus, accurate time estimation as measured by the task of the present study requires both working and reference memories in the memory stage of the SET.

The present results are consistent with those reported by Rao and colleagues [20], who indicated that brain activation in relation to time estimation occurs in the right hemisphere of the cortical network, primarily in the right DLPFC; they found that the right DLPFC was engaged during the comparison of temporal information. Furthermore, we replicated the predominant involvement of the right hemisphere that has been previously observed; patients with right prefrontal cortex lesions show impaired time estimation. Collectively, the evidence demonstrates the importance of right prefrontal cortex activation in time estimation [35].

The results of our study imply that time estimation and working memory depend on the same neural networks. This evidence is consistent with our hypothesis, as the findings generally show that the frontal system is directly responsible for storing temporal information in the memory stage of the SET.

The behavioral results indicated that participants' time estimation was accurate with low variability, indicating that the protocol was successful. These findings are also consistent with previous reports that time estimation is generally accurate when participants can count the required time using conventional time units for the stimulus duration $[3,5,19,36]$.
Coull and colleagues found that attention modulates the subjective perception of time [36]. In single-task paradigms such as the one used in this study, participants focus on the time estimation task itself, whereas paradigms with time estimation and a concurrent task require processing attention to be shared between temporal information and nontemporal information, and fewer pulses are gated into the accumulator and transferred into the working memory in the clock stage of the SET [3]. That is, less attention paid to the temporal properties of a stimulus results in shorter experienced intervals [36]. As silently counting time increases the degree of attention allocated to time, time estimation performance in the present study was generally accurate with minimal variability. In addition, there were no significant differences in the mean values of ratio scores or coefficient of variance indexes among the five time-interval conditions, indicating that time estimation parameters such as accuracy and variability were identical in the range between 3 and $15 \mathrm{~s}$.

The primary contribution of this study is that we were able to use fNIRS to clarify the neural basis of time estimation in seated participants. The results of this study are similar to those of previous fMRI studies. It has been suggested that cognitive function, arousal level, autonomic nervous system function, and fatigue differ between supine and sitting postures (e.g., $[12,16])$. Even though the results of the present study may have been affected by the difference in body posture compared to previous fMRI studies, the same results were obtained using a different brain imaging machine, and irrespective of the body posture, we believe that the present results support previous findings of DLPFC activation during time estimation, indicating that the working memory system is in use. The greatest advantage of fNIRS compared with fMRI is that recordings can be obtained without having to fix the participant's head and body. Because the participants were not excessively restrained, it was possible to obtain measurements while they were in a sitting position, which is advantageous because it is not necessary to consider the effect of body posture on time estimation as required for conventional fMRI in a supine position. Nevertheless, additional studies are needed to further clarify whether this method could be used to assess time estimation in pediatric participants.

Our findings should be interpreted in the context of several limitations. The first is that three trials per time estimation experiment may not have been sufficient to obtain clear results. Other research results indicate that the mechanism 
of time production differs depending on the target duration $[3,37]$. Even though we pooled five time intervals $(3,6,9$, 12 , and $15 \mathrm{~s}$ ) to form a single condition and there were 15 trials in total, this still seems insufficient to overcome the low signal-to-noise ratio in the brain activation signal obtained by fNIRS. Future studies should increase the number of trials per condition to more stringently determine which specific frontal region is involved in timing estimation. The second limitation associated with this study is that we were unable to consider the mutual relationships between brain regions involved in time estimation. fNIRS is a useful technology; however, it can only be used to estimate prefrontal cortex activity. It has recently been suggested that frontal lobe connections through the thalamus and striatum are responsible for time estimation. fMRI studies would be needed to clarify which cortical and subcortical structures, such as the cerebellum and basal ganglia, process and integrate information regarding time estimation. The third limitation of the study was the inability to separate among working memory, reference memory, and attention from timing (or counting) because of the experimental design. In future experiments, a more sophisticated experimental method that prohibits counting would offer better insight into the role of memory and attention in time estimation.

\section{Conclusion}

In conclusion, we used fNIRS to identify frontal brain regions involved in time estimation. Increased activity related to working memory was observed in the right DLPFC when participants were asked to produce accurate time intervals using conventional time units. The results suggest that accurate time estimation and working memory depend on the same neural networks. According to the SET, the memory and comparator stages are considered to be important for producing accurate time intervals. Therefore, the right DLPFC is likely to be the primary cortical region for accurate time estimation in the range of several seconds. Our findings suggest that the right cerebral hemisphere has an advantage over the left with regard to time estimation. fNIRS is a useful modality because it does not require restraint, is relatively tolerant to motion artifacts, and can be effectively employed in psychoneurological investigations of time estimation. This study provides new evidence that further supports the existence of time-related frontal cortex regions. The results contribute to the time estimation literature in a number of ways. We examined the neural substrates of the memory stages of the SET under conditions that have received very little attention. Our study is the first to examine the memory systems and brain structures involved in accurately producing time intervals by sitting participants using fNIRS measurement.

\section{Conflict of Interests}

The authors declare that there is no conflict of interests regarding the publication of this paper.

\section{Acknowledgments}

The authors thank Akifumi Morita, Fumina Tsukiji, Kota Tamai, Kunitake Suzuki, Masanori Kobayashi, and Nobuko Kemmotsu for helpful comments. The authors gratefully acknowledge Ryunosuke Oka for his proficient technical assistance.

\section{References}

[1] M. Wiener, P. Turkeltaub, and H. B. Coslett, "The image of time: a voxel-wise meta-analysis," NeuroImage, vol. 49, no. 2, pp. 17281740, 2010.

[2] U. A. Kumar, A. V. Sangamanatha, and J. Vikas, "Effects of meditation on temporal processing and speech perceptual skills in younger and older adults," Asian Journal of Neuroscience, vol. 2013, Article ID 304057, 8 pages, 2013.

[3] S. Perbal-Hatif, "A neuropsychological approach to time estimation," Dialogues in Clinical Neuroscience, vol. 14, no. 4, pp. 425-432, 2012.

[4] S. Grondin, "Timing and time perception: a review of recent behavioral and neuroscience findings and theoretical directions," Attention, Perception \& Psychophysics, vol. 72, no. 3, pp. 561-582, 2010.

[5] W. H. Meck, "Neuropsychology of timing and time perception," Brain and Cognition, vol. 58, no. 1, pp. 1-8, 2005.

[6] J. Gibbon, "Scalar expectancy theory and Weber's law in animal timing," Psychological Review, vol. 84, no. 3, pp. 279-325, 1977.

[7] J. Gibbon, C. Malapani, C. L. Dale, and C. R. Gallistel, “Toward a neurobiology of temporal cognition: advances and challenges," Current Opinion in Neurobiology, vol. 7, no. 2, pp. 170-184, 1997.

[8] C. V. Buhusi and W. H. Meck, "What makes us tick? Functional and neural mechanisms of interval timing," Nature Reviews Neuroscience, vol. 6, no. 10, pp. 755-765, 2005.

[9] L. Caselli, L. Iaboli, and P. Nichelli, "Time estimation in mild Alzheimer's disease patients," Behavioral and Brain Functions, vol. 5, article 32, 2009.

[10] F. Macar, H. Lejeune, M. Bonnet et al., "Activation of the supplementary motor area and of attentional networks during temporal processing," Experimental Brain Research, vol. 142, no. 4, pp. 475-485, 2002.

[11] P. A. Lewis and R. C. Miall, "Distinct systems for automatic and cognitively controlled time measurement: evidence from neuroimaging," Current Opinion in Neurobiology, vol. 13, no. 2, pp. 250-255, 2003.

[12] R. MacKenzie, C. Sims, R. G. Owens, and A. K. Dixon, "Patients' perceptions of magnetic resonance imaging," Clinical Radiology, vol. 50, no. 3, pp. 137-143, 1995.

[13] M. Muehlhan, U. Lueken, J. Siegert, H.-U. Wittchen, M. N. Smolka, and C. Kirschbaum, "Enhanced sympathetic arousal in response to fMRI scanning correlates with task induced activations and deactivations," PLoS ONE, vol. 8, no. 8, Article ID e72576, 2013.

[14] M. Muehlhan, M. Marxen, J. Landsiedel, H. Malberg, and S. Zaunseder, "The effect of body posture on cognitive performance: a question of sleep quality," Frontiers in Human Neuroscience, vol. 8, article 171, 2014.

[15] H. Rau and T. Elbert, "Psychophysiology of arterial baroreceptors and the etiology of hypertension," Biological Psychology, vol. 57, no. 1-3, pp. 179-201, 2001. 
[16] S. Duschek, N. S. Werner, and G. A. Reyes del Paso, "The behavioral impact of baroreflex function: a review," Psychophysiology, vol. 50, no. 12, pp. 1183-1193, 2013.

[17] Y. Hoshi, "Functional near-infrared optical imaging: utility and limitations in human brain mapping," Psychophysiology, vol. 40, no. 4, pp. 511-520, 2003.

[18] Y. Hoshi, "Functional near-infrared spectroscopy: current status and future prospects," Journal of Biomedical Optics, vol. 12, no. 6, Article ID 062106, 2007.

[19] J. W. Anderson and M. Schmitter-Edgecombe, "Recovery of time estimation following moderate to severe traumatic brain injury," Neuropsychology, vol. 25, no. 1, pp. 36-44, 2011.

[20] S. M. Rao, A. R. Mayer, and D. L. Harrington, "The evolution of brain activation during temporal processing," Nature Neuroscience, vol. 4, no. 3, pp. 317-323, 2001.

[21] C. Fortin and R. Breton, "Temporal interval production and processing in working memory," Perception \& Psychophysics, vol. 57, no. 2, pp. 203-215, 1995.

[22] R. A. Block and R. P. Gruber, "Time perception, attention, and memory: a selective review," Acta Psychologica, vol. 149, pp. 129133, 2014.

[23] A. D. Baddeley, "Working memory: looking back and looking forward," Nature Reviews: Neuroscience, vol. 4, no. 10, pp. 829839, 2003.

[24] R. C. Oldfield, "The assessment and analysis of handedness: the Edinburgh inventory," Neuropsychologia, vol. 9, no. 1, pp. 97-113, 1971.

[25] World Medical Association, "World Medical Association Declaration of Helsinki: ethical principles for medical research involving human subjects," The Journal of the American Medical Association, vol. 310, no. 20, pp. 2191-2194, 2013.

[26] H. H. Jasper, "The ten-twenty electrode system of the International Federation," Electroencephalography and Clinical Neurophysiology, vol. 10, pp. 371-375, 1958.

[27] M.-C. Sévigny, J. Everett, and S. Grondin, "Depression, attention, and time estimation," Brain and Cognition, vol. 53, no. 2, pp. 351-353, 2003.

[28] M. Cope, D. T. Delpy, E. O. Reynolds, S. Wray, J. Wyatt, and P. van der Zee, "Methods of quantitating cerebral near infrared spectroscopy data," Advances in Experimental Medicine and Biology, vol. 222, pp. 183-189, 1988.

[29] M. Sanefuji, Y. Takada, N. Kimura et al., "Strategy in short-term memory for pictures in childhood: a near-infrared spectroscopy study," NeuroImage, vol. 54, no. 3, pp. 2394-2400, 2011.

[30] M. Jueptner and C. Weiller, "Does measurement of regional cerebral blood flow reflect synaptic activity? Implications for PET and fMRI," NeuroImage, vol. 2, no. 2, pp. 148-156, 1995.

[31] K. Sakatani, Y. Murata, N. Fujiwara et al., "Comparison of blood-oxygen-level-dependent functional magnetic resonance imaging and near-infrared spectroscopy recording during functional brain activation in patients with stroke and brain tumors," Journal of Biomedical Optics, vol. 12, no. 6, Article ID 062110, 2007.

[32] R. Aoki, H. Sato, T. Katura et al., "Relationship of negative mood with prefrontal cortex activity during working memory tasks: an optical topography study," Neuroscience Research, vol. 70, no. 2, pp. 189-196, 2011.

[33] Y. Minagawa-Kawai, H. Van Der Lely, F. Ramus, Y. Sato, R. Mazuka, and E. Dupoux, "Optical brain imaging reveals general auditory and language-specific processing in early infant development," Cerebral Cortex, vol. 21, no. 2, pp. 254-261, 2011.
[34] S. W. Greenhouse and S. Geisser, "On methods in the analysis of profile data," Psychometrika, vol. 24, no. 2, pp. 95-112, 1959.

[35] S. Grondin and C. Girard, "About hemispheric differences in the processing of temporal intervals," Brain and Cognition, vol. 58, no. 1, pp. 125-132, 2005.

[36] J. T. Coull, F. Vidal, B. Nazarian, and F. Macar, "Functional anatomy of the attentional modulation of time estimation," Science, vol. 303, no. 5663, pp. 1506-1508, 2004.

[37] G. Koch, M. Oliveri, and C. Caltagirone, "Neural networks engaged in milliseconds and seconds time processing: evidence from transcranial magnetic stimulation and patients with cortical or subcortical dysfunction," Philosophical Transactions of the Royal Society B: Biological Sciences, vol. 364, no. 1525, pp. 1907-1918, 2009. 

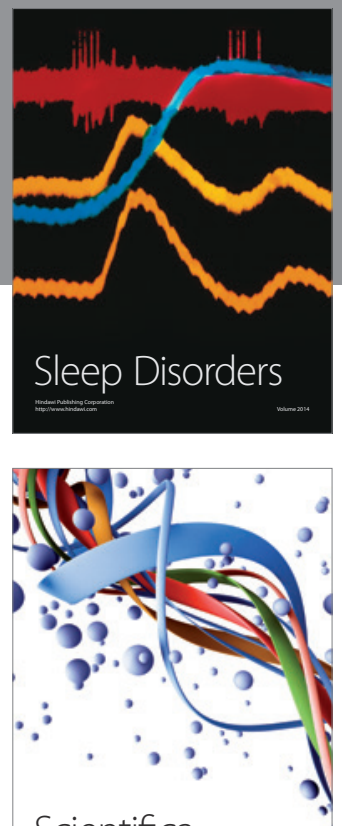

Scientifica
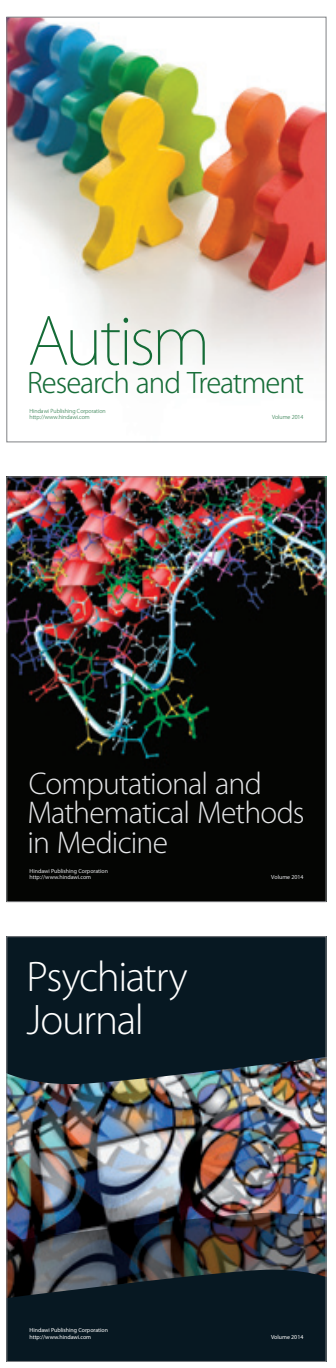
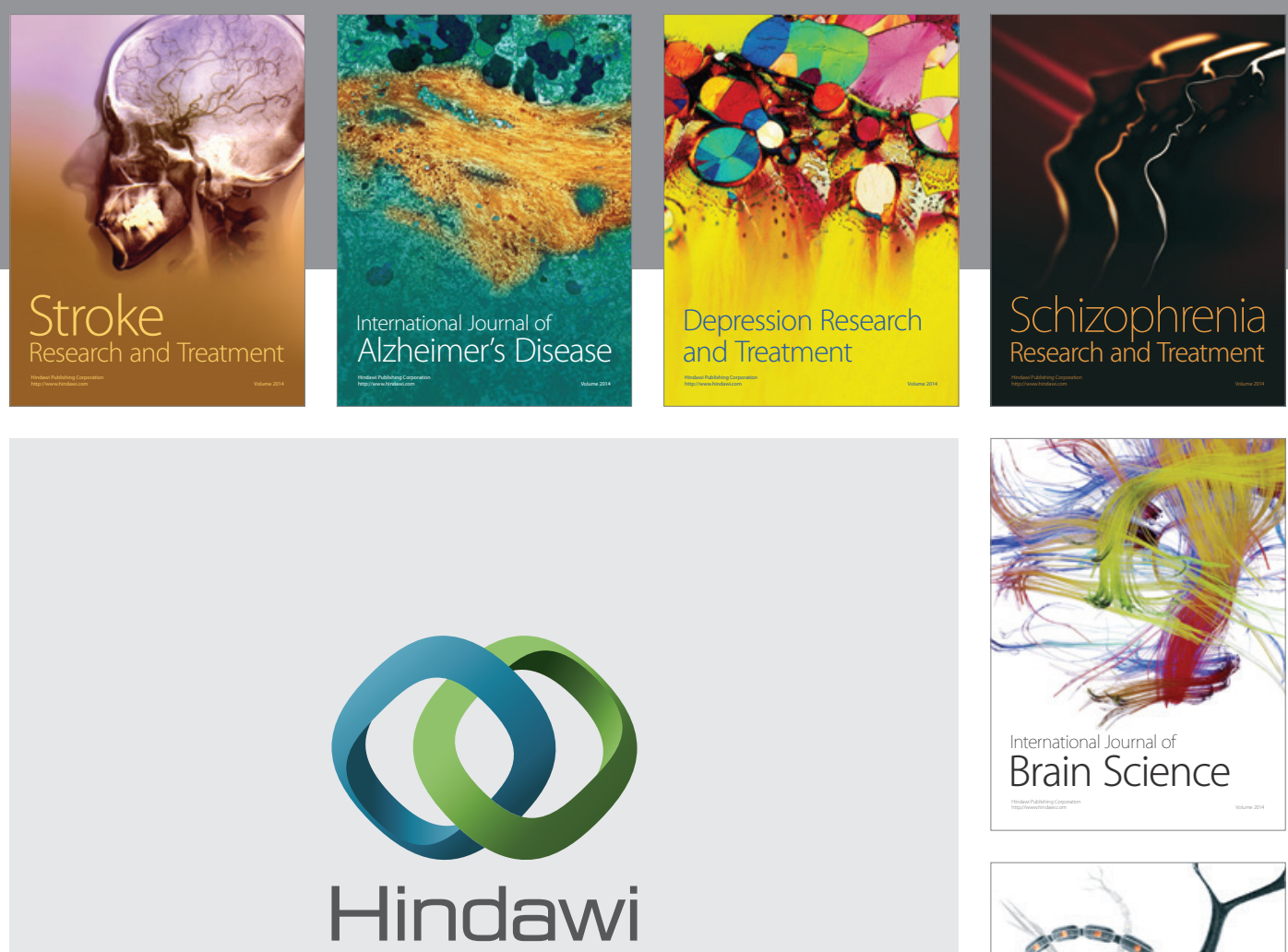

Submit your manuscripts at

http://www.hindawi.com
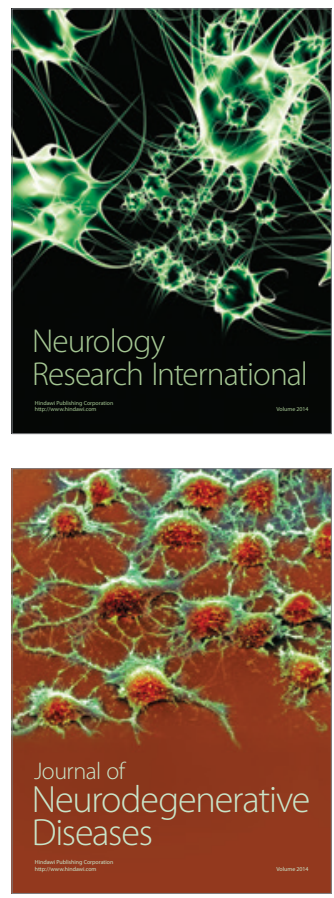

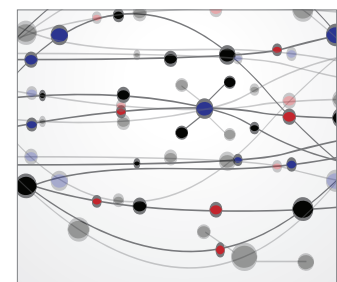

The Scientific World Journal
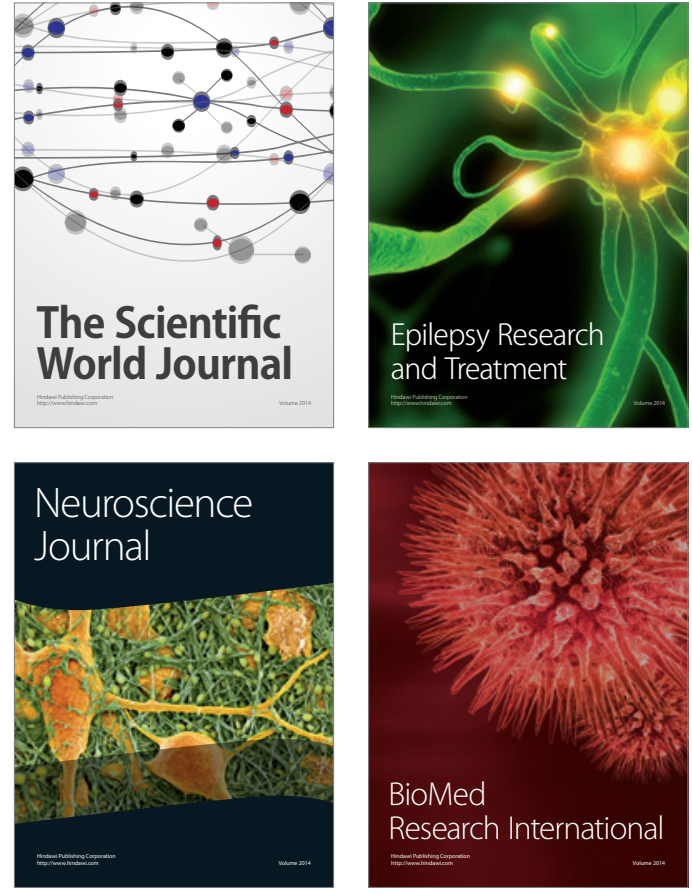

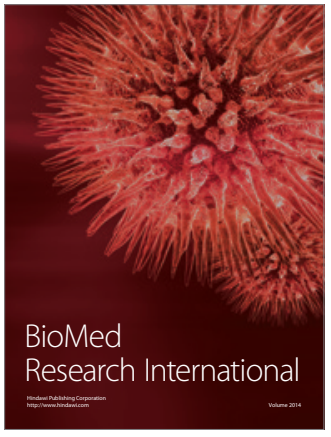

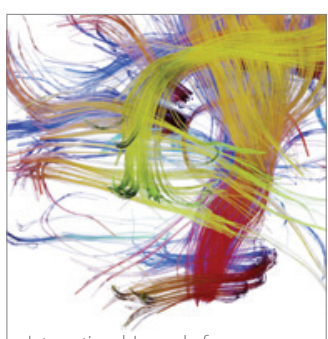

Brain Science

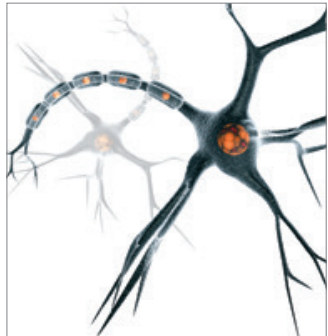

Neural Plasticity
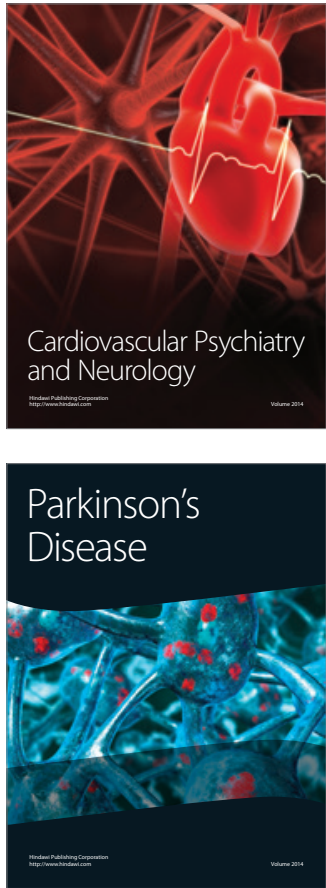